Article

\title{
Existence and Nonexistence of Solutions to $p$-Laplacian Problems on Unbounded Domains
}

\author{
Jeongmi Jeong ${ }^{1}$, Chan-Gyun Kim ${ }^{2, * \mathbb{C}}$ and Eun Kyoung Lee ${ }^{2}$ \\ 1 Department of Mathematics, Pusan National University, Busan 609-735, Korea; jmjeong321@gmail.com \\ 2 Department of Mathematics Education, Pusan National University, Busan 609-735, Korea; eklee@pusan.ac.kr \\ * Correspondence: cgkim75@gmail.com
}

Received: 28 April 2019; Accepted: 14 May 2019; Published: 17 May 2019

check for updates

\begin{abstract}
In this article, using a fixed point index theorem on a cone, we prove the existence and multiplicity results of positive solutions to a one-dimensional $p$-Laplacian problem defined on infinite intervals. We also establish the nonexistence results of nontrivial solutions to the problem.
\end{abstract}

Keywords: $p$-Laplacian; multiplicity of positive solutions; exterior domain

\section{Introduction}

In this paper, we are concerned with the following one-dimensional $p$-Laplacian problem defined on infinite intervals:

$$
\left\{\begin{array}{l}
\frac{1}{r^{N-1}}\left(r^{N-1} \varphi_{p}\left(u^{\prime}\right)\right)^{\prime}+K(r) f(u)=0, \quad r \in(R, \infty) \\
u(R)=\lim _{r \rightarrow \infty} u(r)=0
\end{array}\right.
$$

where $1<p<N, \varphi_{p}(s):=|s|^{p-2} s$ for $s \in \mathbb{R} \backslash\{0\}, \varphi_{p}(0):=0, K \in C^{1}\left(\mathbb{R}_{+}, \mathbb{R}_{+}\right)$with $\mathbb{R}_{+}=(0, \infty)$, $f$ is an odd and locally Lipschitz-continuous function on $\mathbb{R}$, and $R$ is a positive parameter.

Problem (1) arises naturally in the study of radial solutions of nonlinear elliptic equations, with $g(\lambda, x, u)=K(|x|) f(u)$ and $\Omega=\mathbb{R}^{N} \backslash \bar{B}_{R}(0)$, of the form:

$$
\begin{cases}\operatorname{div}\left(|\nabla u|^{p-2} \nabla u\right)+g(\lambda, x, u)=0 & \text { in } \Omega, \\ u=0 & \text { on } \partial \Omega .\end{cases}
$$

For the last several decades, there has been extensive study of Problem (2) with various assumptions for the domain $\Omega$ and the nonlinearity $g=g(\lambda, x, u)$. For example, for $p=2, \Omega=$ $(-1,1)$, and $g(\lambda, x, u)=|x|^{l} u^{p}$, Tanaka ([1]) showed the existence of one positive even solution and two positive non-even solutions to problem (2) when $l(p-1) \geq 4$ and $l \geq 0$. Recently, for $p \in(1, N), \Omega=\mathbb{R}^{N} \backslash \bar{B}_{R}(0)$ and $g(\lambda, x, u)=\lambda K(|x|) f(u)$, Shivaji, Sim, and Son ([2]) proved the uniqueness of positive solution to Problem (2) for large $\lambda$ under suitable additional assumptions on the reaction term $f$ satisfying $f(0)<0$. More recently, for $p \in(1, N), \Omega=\mathbb{R}^{N} \backslash \bar{B}_{1}(0)$ and $g(\lambda, x, u)=\lambda K(x)|u|^{p-2} u+h(x)$, Drábek, Ho, and Sarkar ([3]) investigated the Fredholm alternative for Problem (2) and also discussed the striking difference between the exterior domain and the entire space. For more references, we refer the reader to [4-13] for bounded domains and to [14-23] for unbounded domains.

By a solution $u$ to Problem (1) with $R \in \mathbb{R}_{+}$, we mean $u \in C^{1}(R, \infty) \cap C[R, \infty)$ with $r^{N-1} \varphi_{p}\left(u^{\prime}\right) \in$ $C^{1}(R, \infty)$ satisfies (1). We make a list of hypotheses that are used in this paper. 
(F0) there exist $0 \leq \alpha<\beta \leq \infty$ such that $f(u)<0$ for $u \in(0, \alpha), f(u)>0$ for $u \in(\alpha, \beta)$ and $f(u) \leq 0$ for $u>\beta$;

(F1) there exists $C_{0}>0$ such that $|f(u)| \leq C_{0} u^{p-1}$ for $u \in \mathbb{R}_{+}$;

(F2) $p^{*} K(r)+r K^{\prime}(r) \leq 0$ for $r \in \mathbb{R}_{+}$, where $p^{*}\left(=p^{*}(N, p)\right)=\frac{p(N-1)}{p-1}$;

$(F 2)^{\prime} p^{*} K(r)+r K^{\prime}(r) \geq 0$ for $r \in \mathbb{R}_{+}$;

(F3) $\int_{1}^{\infty}[K(r)]^{\frac{1}{p}} d r<\infty$;

(F4) $\int_{1}^{\infty} r^{p-1} K(r) d r<\infty$;

(F5) there exist positive constants $q_{1}, q_{2}, C_{1}$ and $C_{2}$ such that $p<q_{2} \leq q_{1}, C_{2} r^{-q_{2}} \leq K(r) \leq$ $C_{1} r^{-q_{1}}$ for $r \in\left(0, R_{0}\right)$, and $C_{1} r^{-q_{1}} \leq K(r) \leq C_{2} r^{-q_{2}}$ for $r \in\left(R_{0}, \infty\right)$. Here, $R_{0}=\left(C_{1} C_{2}^{-1}\right)^{\frac{1}{q_{1}-q_{2}}} \in$ $\mathbb{R}_{+}$if $q_{1}>q_{2}$, and $R_{0}=\infty$ if $q_{1}=q_{2}$ and $C_{2} \leq C_{1}$.

\section{Remark 1.}

(1) Note that (F2) (resp., (F2)') holds if and only if $\frac{d}{d r}\left[r^{*} K(r)\right] \leq 0$ (resp., $\geq 0$ ) for $r \in \mathbb{R}_{+}$.

(2) Assume that, for some constants $C$ and $q, K(r)=C r^{-q}$ for $r \in \mathbb{R}_{+}$. Then, (F2) holds if $q \geq p^{*}$; $(F 2)^{\prime}$ holds if $q \leq p^{*}$; (F3) and (F4) hold if $q>p$. Since $p<N<p^{*},(F 2),(F 3)$, and (F4) hold if $q \geq p^{*} ;(F 2)^{\prime},(F 3)$, and (F4) hold if $p<q \leq p^{*}$. Note that, for any $R \in \mathbb{R}_{+}, q>N$ if and only if $r^{N-1} K(r) \in L^{1}(R, \infty)$. Thus, in this case, (F2) implies $r^{N-1} K(r) \in L^{1}(R, \infty)$, but $r^{N-1} K(r)$ may not be in $L^{1}(R, \infty)$ if $(F 2)^{\prime}$ holds.

(3) (F5) implies (F3) and (F4).

This paper is motivated by the recent works of Iaia ([16-19]), Joshi ([24]), and Joshi and Iaia ([20]). For $p=2$, the existence of an infinite number of solutions with a prescribed number of zeros to Problem (1) was proven in [16-20,24], and the nonexistence of nontrivial solutions to Problem (1) was shown in $[16,18,24]$. The proofs in those papers are mainly based on the shooting method. In this paper, for $p \in(1, N)$, the nonexistence results of nontrivial solutions to Problem (1) are proven for sufficiently large $R>0$, and the existence results of positive solutions to Problem (1) are established. Our approach for the existence results of positive solutions is based on a fixed point index theorem on positive cones.

\section{Nonexistence of Nontrivial Solutions to Problem (1)}

Let $u$ be a solution to Problem (1). Then, it is well known that $u \in C^{2}([R, \infty))$ for $p \in(1,2]$ and $u \in C^{1}([R, \infty)) \cap C^{2}([R, \infty) \backslash A)$ for $p>2$, where $A=\left\{r \in[R, \infty): u^{\prime}(r)=0\right\}$. Clearly, zero is a trivial solution to Problem (1), since $f(0)=0$.

Theorem 1. Assume that (F0), (F1), (F2), and (F3) hold. Then, there exists $R_{*}>0$ such that Problem (1) has no nontrivial solutions for any $R>R_{*}$.

Proof. Assume, on the contrary, that there exists a nontrivial solution $u$ to Problem (1). By (F1), if $u^{\prime}(R)=0$, then $u \equiv 0$ on $[R, \infty)$ in view of [12] (Theorem $4(\delta)$ ). Thus, $u^{\prime}(R) \neq 0$. We may assume that $u^{\prime}(R)>0$, since $f$ is an odd function. Then, there exists $M_{1}>R$ such that $u^{\prime}(r)>0$ for $\left[R, M_{1}\right)$ and $u^{\prime}\left(M_{1}\right)=0$. Set:

$$
E_{1}[u](r)=\frac{p-1}{p} \frac{\left|u^{\prime}(r)\right|^{p}}{K(r)}+F(u(r)) \text { for } r \in[R, \infty),
$$

where $F(v)=\int_{0}^{v} f(s) d s$ for $v \in \mathbb{R}$. Since $u \in C^{2}\left(\left[R, M_{1}\right)\right)$ is a solution to Problem (1),

$$
\frac{d}{d r} E_{1}[u](r)=-\frac{(p-1)\left|u^{\prime}(r)\right|^{p}}{p r(K(r))^{2}}\left[p^{*} K(r)+r K^{\prime}(r)\right] \text { for } r \in\left[R, M_{1}\right) .
$$


By $(F 2), \frac{d}{d r} E_{1}[u](r) \geq 0$ for $r \in\left[R, M_{1}\right)$. Consequently, $E_{1}[u]$ is nondecreasing in $\left[R, M_{1}\right)$, and:

$$
E_{1}[u](r) \leq E_{1}[u]\left(M_{1}\right)=F\left(u\left(M_{1}\right)\right) \text { for } r \in\left[R, M_{1}\right],
$$

which implies $F(u(r))<F\left(u\left(M_{1}\right)\right)$ for $r \in\left[R, M_{1}\right]$. In particular, $0=F(u(R))<F\left(u\left(M_{1}\right)\right)$. By (F0), there exist $\gamma \in[\alpha, \beta)$ and $\delta \in[\beta, \infty]$ such that $F(u)<0$ for $u \in(0, \gamma), F(u)>0$ for $u \in(\gamma, \delta)$ and $F(u) \leq 0$ for $u>\delta$. Here, $\gamma=0$ if $\alpha=0$, and $\gamma \in(\alpha, \delta)$ if $\alpha>0$. Assume first that $\alpha>0$. Since $F\left(u\left(M_{1}\right)\right)>0, u\left(M_{1}\right) \in(\gamma, \delta)$, and thus, there exists a constant $F_{0}>0$ satisfying:

$$
F(t)>-F_{0} \text { for all } t \in\left(0, u\left(M_{1}\right)\right] .
$$

By (3) and (4), $[K(r)]^{\frac{1}{p}} \geq \sqrt[p]{\frac{p-1}{p}} \frac{u^{\prime}(r)}{\sqrt[p]{F\left(u\left(M_{1}\right)+F_{0}\right.}}$ for $r \in\left[R, M_{1}\right]$. Then:

$$
\begin{aligned}
\int_{R}^{\infty}[K(r)]^{\frac{1}{p}} d r & \geq \int_{R}^{M_{1}}[K(r)]^{\frac{1}{p}} d r \geq \sqrt[p]{\frac{p-1}{p}} \int_{R}^{M_{1}} \frac{u^{\prime}(r)}{\sqrt[p]{F\left(u\left(M_{1}\right)\right)+F_{0}}} d r \\
& =\sqrt[p]{\frac{p-1}{p}}\left[\sqrt[p]{\frac{F\left(u\left(M_{1}\right)\right)}{\left[u\left(M_{1}\right)\right]^{p}}+\frac{F_{0}}{\left[u\left(M_{1}\right)\right]^{p}}}\right]^{-1} .
\end{aligned}
$$

By $(F 1)$ and from the fact $u\left(M_{1}\right) \in(\gamma, \delta)$, it follows that:

$$
0<\frac{F\left(u\left(M_{1}\right)\right)}{\left[u\left(M_{1}\right)\right]^{p}}+\frac{F_{0}}{\left[u\left(M_{1}\right)\right]^{p}} \leq \frac{C_{0}}{p}+\frac{F_{0}}{\gamma^{p}} .
$$

Consequently,

$$
0<\sqrt[p]{\frac{p-1}{p}}\left[\sqrt[p]{\frac{C_{0}}{p}+\frac{F_{0}}{\gamma^{p}}}\right]^{-1} \leq \int_{R}^{\infty}[K(r)]^{\frac{1}{p}} d r .
$$

By (F3), $\int_{R}^{\infty}[K(r)]^{\frac{1}{p}} d r \rightarrow 0$ as $R \rightarrow \infty$, and thus, there exists $R_{*}>0$ such that Problem (1) has no nontrivial solution for any $R>R_{*}$. Next, assume that $\alpha=0$. Then, $u\left(M_{1}\right) \in(0, \delta)$, and (4) with $F_{0}=0$ holds. Then, one can easily prove the desired result, by arguments similar to those in the proof for the case $\alpha>0$. Thus, the proof is complete.

Using transformation $v(t)=u(r)$ with $t=r^{\frac{p-N}{p-1}}$, (1) can be rewritten equivalently as follows:

$$
\left\{\begin{array}{l}
\left(\varphi_{p}\left(v^{\prime}\right)\right)^{\prime}+h(t) f(v)=0, \quad t \in\left(0, R^{\frac{p-N}{p-1}}\right), \\
v(0)=v\left(R^{\frac{p-N}{p-1}}\right)=0,
\end{array}\right.
$$

where $h(t)=\left(\frac{p-1}{N-p}\right)^{p} t^{\frac{p(N-1)}{p-N}} K\left(t^{\frac{p-1}{p-N}}\right)=\left(\frac{p-1}{N-p}\right)^{p} r^{p^{*}} K(r)$.

For the sake of convenience, we make a list of classes of the weight $h$ as follows:

- $\mathcal{A}=\left\{h \in C\left(\mathbb{R}_{+}, \mathbb{R}_{+}\right): \int_{0}^{1} s^{p-1} h(s) d s<\infty\right\} ;$

- $\mathcal{B}=\left\{h \in C\left(\mathbb{R}_{+}, \mathbb{R}_{+}\right): \int_{0}^{1} \varphi_{p}^{-1}\left(\int_{s}^{1} h(\tau) d \tau\right) d s<\infty\right\} ;$

- $\mathcal{C}=\left\{h \in C\left(\mathbb{R}_{+}, \mathbb{R}_{+}\right): \int_{0}^{1} s^{\theta} h(s) d s<\infty\right.$ for some $\left.\theta \in(0, p-1)\right\}$;

- $\mathcal{D}=\left\{h \in C\left(\mathbb{R}_{+}, \mathbb{R}_{+}\right): \int_{0}^{1}[h(s)]^{\frac{1}{p}} d s<\infty\right\}$. 


\section{Remark 2.}

(1) It is well known that $L^{1}(0,1) \cap \mathcal{C}\left(\mathbb{R}_{+}, \mathbb{R}_{+}\right) \subsetneq \mathcal{C} \subsetneq \mathcal{A} \cap \mathcal{B}, \mathcal{A} \subsetneq \mathcal{B}$ for $p \in(1,2), \mathcal{B} \subsetneq \mathcal{A}$ for $p>2$, and $\mathcal{A}=\mathcal{B}$ for $p=2$ (see, e.g., [25]). It is obvious that $h \in \mathcal{C}$, provided $h(t)=t^{-\rho}$ for some $\rho<p$. Moreover, $\mathcal{C} \subseteq \mathcal{D}$. Indeed, let $h \in \mathcal{C}$, and let $p^{\prime}:=\frac{p}{p-1} \in(1, \infty)$. Then, $\theta\left(-\frac{p^{\prime}}{p}\right)>-1$, and by the Hölder inequality,

$$
\int_{0}^{1}[h(t)]^{\frac{1}{p}} d t=\int_{0}^{1}\left(t^{\theta} h(t)\right)^{\frac{1}{p}}\left(t^{\theta}\right)^{-\frac{1}{p}} d t \leq\left[\int_{0}^{1} t^{\theta} h(t) d t\right]^{\frac{1}{p}}\left[\int_{0}^{1}\left(t^{\theta}\right)^{-\frac{p^{\prime}}{p}} d t\right]^{\frac{1}{p^{\prime}}}<\infty .
$$

(2) Assume that $h(t)=\left(\frac{p-1}{N-p}\right)^{p} r^{p^{*}} K(r)$ with $t=r^{\frac{p-N}{p-1}}$. Then, (F3) holds if and only if $h \in \mathcal{D}$, and (F4) holds if and only if $h \in \mathcal{A}$.

(3) In [6] (Theorem 2.4), the $C^{1}$-regularity of solutions was proven, provided $h \in \mathcal{A} \cap \mathcal{B}$ and $f(s)>0$ for $s>0$. However, if $p>2$ and $h \in \mathcal{A} \backslash \mathcal{B}$, the solutions to (5) may not be in $C^{1}\left[0, R^{\frac{p-N}{p-1}}\right]$ (see, e.g., [6] (Example 2.7)).

Lemma 1. Assume that (F0), (F1) and (F4) hold. Let $v$ be a nontrivial solution to (5). Then, there exists $\delta>0$ such that $v(t) \neq 0$ for $t \in(0, \delta)$, and $v^{\prime}(0) \in[-\infty, 0) \cup(0, \infty]$.

Proof. Let $v$ be a nontrivial solution to Problem (5). Then, $v \in C\left[0, R^{\frac{p-N}{p-1}}\right] \cap C^{1}\left(0, R^{\frac{p-N}{p-1}}\right]$. First, we prove that there exists $\delta>0$ such that $v(t) \neq 0$ for $t \in(0, \delta)$. Assume, on the contrary, that there exists a strictly decreasing sequence $\left(t_{n}\right)$ satisfying $v\left(t_{n}\right)=0$ and $t_{n} \rightarrow 0$ as $n \rightarrow \infty$. Multiplying the first equation in (5) by $v$ and integrating it over $\left(t_{n+1}, t_{n}\right)$, by $(F 1)$,

$$
\int_{t_{n+1}}^{t_{n}}\left|v^{\prime}(s)\right|^{p} d s=\int_{t_{n+1}}^{t_{n}} h(s) f(v(s)) v(s) d s \leq C_{0} \int_{t_{n+1}}^{t_{n}} h(s)|v(s)|^{p} d s .
$$

By the Hölder inequality, for $s \in\left(t_{n+1}, t_{n}\right)$,

$$
|v(s)| \leq \int_{t_{n+1}}^{s}\left|v^{\prime}(\tau)\right| d \tau \leq s^{\frac{p-1}{p}}\left(\int_{t_{n+1}}^{s}\left|v^{\prime}(\tau)\right|^{p} d \tau\right)^{\frac{1}{p}}
$$

Substituting (7) into the integrand on the right-hand side in (6),

$$
\int_{t_{n+1}}^{t_{n}}\left|v^{\prime}(s)\right|^{p} d s \leq C_{0} \int_{t_{n+1}}^{t_{n}} s^{p-1} h(s) d s \int_{t_{n+1}}^{t_{n}}\left|v^{\prime}(s)\right|^{p} d s .
$$

By $(F 4), h \in \mathcal{A}$, and there exists $N>0$ such that $C_{0} \int_{0}^{t_{N}}{ }_{s}^{p-1} h(s) d s<\frac{1}{2}$. For any $n \geq N$,

$$
\int_{t_{n+1}}^{t_{n}}\left|v^{\prime}(s)\right|^{p} d s<\frac{1}{2} \int_{t_{n+1}}^{t_{n}}\left|v^{\prime}(s)\right|^{p} d s .
$$

which implies $v^{\prime}(s)=0$ for $s \in\left[t_{n+1}, t_{n}\right]$ for all $n \geq N$. Consequently, by $(7), v \equiv 0$ on $\left[0, t_{N}\right]$. Since $h \in C\left[t_{N}, R^{\frac{p-N}{p-1}}\right]$, by Grönwall's inequality, it can be easily proven that $v \equiv 0$ on $\left[0, R^{\frac{p-N}{p-1}}\right]$, which contradicts the fact that $v$ is a nontrivial solution to Problem (5).

Since $f$ is an odd function, we may assume that, for some $\delta>0, v(t)>0$ for $t \in(0, \delta)$. We prove $v^{\prime}(0) \in(0, \infty]$ in order to complete the proof. Since $v$ is a solution to Problem (5), by $(F 0), v^{\prime}$ is a monotonic function in $\left(0, \delta_{1}\right)$ for some $\delta_{1} \in(0, \delta)$. Then, $\lim _{t \rightarrow 0+} v^{\prime}(t) \in[0, \infty]$. Assume, on the contrary, that $v^{\prime}(0)=0$. By L'Hôpital's rule, $v^{\prime}(0)=\lim _{t \rightarrow 0+} \frac{v(t)}{t}=\lim _{t \rightarrow 0+} v^{\prime}(t)$, and thus, $v \in C^{1}\left[0, R^{\frac{p-N}{p-1}}\right]$ 
satisfying $v^{\prime}(0)=0$. Define $w:\left[0, R^{\frac{p-N}{p-1}}\right] \rightarrow \mathbb{R}$ by $w(0)=0$ and $w(t)=\frac{v(t)}{t}$ for $t \in\left(0, R^{\frac{p-N}{p-1}}\right]$. Then $w \in C\left[0, R^{\frac{p-N}{p-1}}\right]$, since $v^{\prime}(0)=0$. For $t \in\left(0, R^{\frac{p-N}{p-1}}\right]$,

$$
\begin{aligned}
|w(t)| & =\left|\frac{1}{t} \int_{0}^{t} \varphi_{p}^{-1}\left(\int_{0}^{s} h(\tau) f(v(\tau)) d \tau\right) d s\right| \leq \frac{1}{t} \int_{0}^{t} \varphi_{p}^{-1}\left(\int_{0}^{s} h(\tau)|f(v(\tau))| d \tau\right) d s \\
& \leq \varphi_{p}^{-1}\left(\int_{0}^{t} h(\tau)|f(v(\tau))| d \tau\right) .
\end{aligned}
$$

By $(F 1),|w(t)|^{p-1} \leq C_{0} \int_{0}^{t} h(\tau)|v(\tau)|^{p-1} d \tau=C_{0} \int_{0}^{t} \tau^{p-1} h(\tau)|w(\tau)|^{p-1} d \tau$ for $t \in\left(0, R^{\frac{p-N}{p-1}}\right]$. By Grönwall's inequality, $|w(t)|^{p-1}=0$ on $\left[0, R^{\frac{p-N}{p-1}}\right]$, and consequently, $v \equiv 0$ on $\left[0, R^{\frac{p-N}{p-1}}\right]$, which contradicts the fact that $v$ is a nontrivial solution to problem (5). Thus, the proof is complete.

Theorem 2. Assume that (F0), (F1), (F2)', (F3), and (F4) hold. Then, there exists $R_{*}>0$ such that for any $R>R_{*}$, Problem (5) (or equivalently (1)) has no nontrivial solutions.

Proof. Let $v$ be a nontrivial solution to Problem (5). Then, $v \in C\left[0, R^{\frac{p-N}{p-1}}\right] \cap C^{1}\left(0, R^{\frac{p-N}{p-1}}\right]$. By Lemma 1 , we may assume that $v^{\prime}(0) \in(0, \infty]$ and $v(t)>0$ for $t \in(0, \delta)$, since $f$ is an odd function. Then, there exists $M_{2} \in\left(0, R^{\frac{p-N}{p-1}}\right)$ such that $v^{\prime}(t)>0$ for $\left(0, M_{2}\right)$ and $v^{\prime}\left(M_{2}\right)=0$. Thus, $v \in C^{2}\left(0, M_{2}\right)$. Set:

$$
E_{2}[v](t)=\frac{p-1}{p} \frac{\left|v^{\prime}(t)\right|^{p}}{h(t)}+F(v(t)), t \in\left(0, R^{\frac{p-N}{p-1}}\right] .
$$

From the facts $\frac{d r}{d t}=\frac{p-1}{p-N} \frac{r}{t}<0$ and $\frac{d h}{d t}=\left(\frac{p-1}{N-p}\right)^{p} \frac{d}{d r}\left[r^{p^{*}} K(r)\right] \frac{d r}{d t}$, it follows that $(F 2)^{\prime}$ holds if and only if $h^{\prime} \leq 0$ on $\mathbb{R}_{+}$. Since $v \in C^{2}\left(0, M_{2}\right)$, by $(F 2)^{\prime}$,

$$
\frac{d}{d t} E_{2}[v](t)=-\frac{p-1}{p} \frac{\left|v^{\prime}(t)\right|^{p} h^{\prime}(t)}{[h(t)]^{2}} \geq 0 \text { for } t \in\left(0, M_{2}\right),
$$

and thus, $E_{2}[v]$ is nondecreasing in $\left(0, M_{2}\right)$. By an argument similar to those in the proof of Theorem 1 , the proof is complete.

\section{Existence of Positive Solutions to Problem (1)}

Let $R \in \mathbb{R}_{+}$be given. Using transformation $w(t)=u(r)$ with $t=\left(R^{-1} r\right)^{\frac{p-N}{p-1}},(1)$ can be rewritten equivalently as follows:

$$
\left\{\begin{array}{l}
\left(\varphi_{p}\left(w^{\prime}\right)\right)^{\prime}+h_{R}(t) f(w)=0, t \in(0,1), \\
w(0)=w(1)=0
\end{array}\right.
$$

where $h_{R}(t)=\left(\frac{p-1}{N-p}\right)^{p} R^{p} t^{\frac{p(N-1)}{p-N}} K\left(R t^{\frac{p-1}{p-N}}\right) \in C\left((0,1], \mathbb{R}_{+}\right)$.

For convenience, we denote ( $F 0)$ with $\alpha=0$ and $\beta=\infty$ by $(F 0)^{\prime}$, i.e.,

$(F 0)^{\prime} f(u)>0$ for $u \in(0, \infty)$.

Throughout this section, we assume $(F 0)^{\prime}$ and $(F 5)$ hold, unless otherwise stated.

Remark 3. Assume that (F5) holds and that $R \in\left(0, R_{0}\right)$. Then, (F5) implies that:

$$
C_{2} R^{p-q_{2}} k_{1}(t) \leq h_{R}(t) \leq C_{1} R^{p-q_{1}} k_{2}(t) \text { for } t \in(0,1],
$$

where $k_{i}(t)=\left(\frac{p-1}{N-p}\right)^{p} t^{\frac{p(N-1)-q_{i}(p-1)}{p-N}}$ for $i=1$, 2. Since $p<q_{2} \leq q_{1}$, 
so that $h_{R} \in \mathcal{C} \subsetneq \mathcal{A} \cap \mathcal{B} \cap \mathcal{D}$.

$$
\frac{p(N-1)-q_{1}(p-1)}{p-N} \geq \frac{p(N-1)-q_{2}(p-1)}{p-N}>-p,
$$

Denote $X=\left(C_{0}[0,1],\|\cdot\|_{\infty}\right)$, where $C_{0}[0,1]=\{w \in C[0,1]: w(0)=w(1)=0\}$ and $\|w\|_{\infty}=$ $\max _{t \in[0,1]}|w(t)|$ for $w \in \mathcal{C}_{0}[0,1]$. Then, $X$ is a Banach space, and $\mathcal{K}=\{w \in X: w$ is a nonnegative and concave function $\}$ is a positive cone in $X$. For $r>0$, we define $\mathcal{K}_{r}=\left\{w \in \mathcal{K}:\|w\|_{\infty}<r\right\}$, $\partial \mathcal{K}_{r}=\left\{w \in \mathcal{K}:\|w\|_{\infty}=r\right\}$, and $\overline{\mathcal{K}}_{r}=\mathcal{K}_{r} \cup \partial \mathcal{K}_{r}$. For $w \in \mathcal{K}$, it is well known that, for any $\delta \in(0,1 / 2), w(t) \geq \delta\|w\|_{\infty}$ for all $t \in[\delta, 1-\delta]$ by the concavity of $w$ on $[0,1]$ (see, e.g., [26] (Lemma 1$)$ ).

The following well-known result on the fixed point index is crucial in this section:

Lemma 2 ([27,28]). Assume that, for some $r>0, T: \overline{\mathcal{K}}_{r} \rightarrow \mathcal{K}$ is completely continuous, i.e., compact and continuous on $\overline{\mathcal{K}}_{r}$. Then, the following results hold:

(i) if $\|T x\|_{\infty}>\|x\|_{\infty}$ for $x \in \partial \mathcal{K}_{r}$, then $i\left(T, \mathcal{K}_{r}, \mathcal{K}\right)=0$;

(ii) if $\|T x\|_{\infty}<\|x\|_{\infty}$ for $x \in \partial \mathcal{K}_{r}$, then $i\left(T, \mathcal{K}_{r}, \mathcal{K}\right)=1$.

Let $R \in \mathbb{R}_{+}$be given. Define $T_{R}: \mathcal{K} \rightarrow \mathcal{K}$ by, for $w \in \mathcal{K}$,

$$
T_{R}(w)(t)= \begin{cases}\int_{0}^{t} \varphi_{p}^{-1}\left(\int_{s}^{A_{R, w}} h_{R}(\tau) f(w(\tau)) d \tau\right) d s, & 0 \leq t \leq A_{R, w}, \\ \int_{t}^{1} \varphi_{p}^{-1}\left(\int_{A_{R, w}}^{s} h_{R}(\tau) f(w(\tau)) d \tau\right) d s, & A_{R, w} \leq t \leq 1,\end{cases}
$$

where $A_{R, w}$ is a constant satisfying:

$$
\int_{0}^{A_{R, w}} \varphi_{p}^{-1}\left(\int_{s}^{A_{R, w}} h_{R}(\tau) f(w(\tau)) d \tau\right) d s=\int_{A_{R, w}}^{1} \varphi_{p}^{-1}\left(\int_{A_{R, w}}^{s} h_{R}(\tau) f(w(\tau)) d \tau\right) d s .
$$

Since $h_{R} \in \mathcal{B}$, it is well known that $T_{R}$ is well defined, $T_{R}(\mathcal{K}) \subseteq \mathcal{K}$, and $T_{R}$ is completely continuous on $\mathcal{K}$ (see, e.g., [4] (Lemma 3)). Clearly, $T_{R}\left(A_{R, w}\right)=\left\|T_{R}(w)\right\|_{\infty}$ for all $R \in \mathbb{R}_{+}$and all $w \in \mathcal{K}$. It can be easily seen that (8) has a positive solution $w$ if and only if $T_{R}$ has a fixed point $w$ in $\mathcal{K} \backslash\{0\}$.

Let $f(m)=\min \left\{f(y): \frac{1}{4} m \leq y \leq m\right\}$ and $\bar{f}(m)=\max \{f(y): 0 \leq y \leq m\}$ for $m \in \mathbb{R}_{+}$. Define continuous functions $R_{1}, R_{2}: \mathbb{R}_{+} \rightarrow \mathbb{R}_{+}$by:

Here:

$$
R_{1}(m)=\frac{m^{p-1}}{\underline{f}(m) A_{1}^{p-1}} \text { and } R_{2}(m)=\frac{m^{p-1}}{\bar{f}(m) A_{2}^{p-1}} \text { for } m \in \mathbb{R}_{+} .
$$

$$
A_{1}=\min \left\{\int_{\frac{1}{4}}^{\frac{1}{2}} \varphi_{p}^{-1}\left(\int_{s}^{\frac{1}{2}} k_{1}(\tau) d \tau\right) d s, \int_{\frac{1}{2}}^{\frac{3}{4}} \varphi_{p}^{-1}\left(\int_{\frac{1}{2}}^{s} k_{1}(\tau) d \tau\right) d s\right\}
$$

and:

$$
A_{2}=\max \left\{\int_{0}^{\frac{1}{2}} \varphi_{p}^{-1}\left(\int_{s}^{\frac{1}{2}} k_{2}(\tau) d \tau\right) d s, \int_{\frac{1}{2}}^{1} \varphi_{p}^{-1}\left(\int_{\frac{1}{2}}^{s} k_{2}(\tau) d \tau\right) d s\right\} .
$$

Remark 4. It is easily verified that $(\bar{f})_{c}=0$ if $f_{c}=0$, and $(\underline{f})_{c}=\infty$ if $f_{c}=\infty$. Here, $f_{c}:=\lim _{s \rightarrow+c} \frac{f(s)}{s^{p-1}}$ for $c \in\{0, \infty\}$. Consequently, $\lim _{m \rightarrow+c} R_{2}(m)=\infty$ if $f_{c}=0$, and $\lim _{m \rightarrow+c} R_{1}(m)=0$ if $f_{c}=\infty$. Since $R_{1}(m)>R_{2}(m)>0$ for all $m \in \mathbb{R}_{+}$, for $i=1,2, \lim _{m \rightarrow+c} R_{i}(m)=\infty$ if $f_{c}=0$, and $\lim _{m \rightarrow+c} R_{i}(m)=0$ if $f_{c}=\infty$ for $c \in\{0, \infty\}$.

Lemma 3. Assume that $(F 0)^{\prime}$ and (F5) hold. Let $m \in \mathbb{R}_{+}$be fixed. Then, for any $R \in\left(0, R_{0}\right)$ satisfying $C_{2} R^{p-q_{2}}>R_{1}(m)$,

$$
i\left(T_{R}, \mathcal{K}_{m}, \mathcal{K}\right)=0
$$


Proof. Let $R \in\left(0, R_{0}\right)$ satisfying $C_{2} R^{p-q_{2}}>R_{1}(m)$ be fixed, and let $w \in \partial \mathcal{K}_{m}$. Then, $\frac{1}{4} m \leq w(t) \leq m$ for $t \in\left[\frac{1}{4}, \frac{3}{4}\right]$, and:

$$
f(w(t)) \geq \underline{f}(m)=\frac{m^{p-1}}{R_{1}(m) A_{1}^{p-1}} \text { for } t \in\left[\frac{1}{4}, \frac{3}{4}\right] .
$$

We have two cases: either $(i) A_{R, w} \in\left[\frac{1}{2}, 1\right)$ or $(i i) A_{R, w} \in\left(0, \frac{1}{2}\right)$. We only consider the case $(i)$, since the case (ii) can be dealt with in a similar manner. Since $C_{2} R^{p-q_{2}}>R_{1}(m)$, from (9) and (11), it follows that:

$$
\begin{aligned}
\left\|T_{R}(w)\right\|_{\infty} & =T_{R}(w)\left(A_{R, w}\right)=\int_{0}^{A_{R, w}} \varphi_{p}^{-1}\left(\int_{s}^{A_{R, w}} h_{R}(\tau) f(w(\tau)) d \tau\right) d s \\
& \geq \int_{\frac{1}{4}}^{\frac{1}{2}} \varphi_{p}^{-1}\left(\int_{s}^{\frac{1}{2}} C_{2} R^{p-q_{2}} k_{1}(\tau) \frac{m^{p-1}}{R_{1}(m) A_{1}^{p-1}} d \tau\right) d s \\
& >\frac{m}{A_{1}} \int_{\frac{1}{4}}^{\frac{1}{2}} \varphi_{p}^{-1}\left(\int_{s}^{\frac{1}{2}} k_{1}(\tau) d \tau\right) d s \geq m=\|w\|_{\infty} .
\end{aligned}
$$

By Lemma 2, (10) holds for any $R \in\left(0, R_{0}\right)$ satisfying $C_{2} R^{p-q_{2}}>R_{1}(m)$. Thus, the proof is complete.

Lemma 4. Assume that (F0)' and (F5) hold. Let $m \in \mathbb{R}_{+}$be fixed. Then, for any $R \in\left(0, R_{0}\right)$ satisfying $C_{1} R^{p-q_{1}}<R_{2}(m)$,

$$
i\left(T_{R}, \mathcal{K}_{m}, \mathcal{K}\right)=1
$$

Proof. Let $R \in\left(0, R_{0}\right)$ satisfying $C_{1} R^{p-q_{1}}<R_{2}(m)$ be fixed, and let $w \in \partial \mathcal{K}_{m}$. Then:

$$
f(w(t)) \leq \bar{f}(m)=\frac{m^{p-1}}{R_{2}(m) A_{2}^{p-1}} \text { for } t \in[0,1] .
$$

We only consider $A_{R, w} \in\left(0, \frac{1}{2}\right)$, since the case $A_{R, w} \in\left[\frac{1}{2}, 1\right)$ can be dealt with in a similar manner. Since $C_{1} R^{p-q_{1}}<R_{2}(m)$, from (9) and (13), it follows that:

$$
\begin{aligned}
\left\|T_{R}(w)\right\|_{\infty} & =T_{R}(u)\left(A_{R, w}\right)=\int_{0}^{A_{R, u}} \varphi_{p}^{-1}\left(\int_{s}^{A_{R, u}} h_{R}(\tau) f(w(\tau)) d \tau\right) d s \\
& \leq \int_{0}^{\frac{1}{2}} \varphi_{p}^{-1}\left(\int_{s}^{\frac{1}{2}} C_{1} R^{p-q_{1}} k_{2}(\tau) \frac{m^{p-1}}{R_{2}(m) A_{2}^{p-1}} d \tau\right) d s \\
& <\frac{m}{A_{2}} \int_{0}^{\frac{1}{2}} \varphi_{p}^{-1}\left(\int_{s}^{\frac{1}{2}} k_{2}(\tau) d \tau\right) d s \leq m=\|w\|_{\infty} .
\end{aligned}
$$

By Lemma 2, (12) holds for any $R \in\left(0, R_{0}\right)$ satisfying $C_{1} R^{p-q_{1}}<R_{2}(m)$, and thus, the proof is complete.

By Lemmas 3 and 4, the result that (8) (or equivalently (1)) has arbitrarily many positive solutions can be obtained. For example, we have the following Theorems 3-8. Since the proofs are similar, we only give the proof of Theorem 6 in detail.

Theorem 3. Assume that (F0)' and (F5) and that there exist $R \in\left(0, R_{0}\right), m_{1}$, and $m_{2}$ such that $0<m_{1}<m_{2}$ (resp., $\left.0<m_{2}<m_{1}\right), C_{2} R^{p-q_{2}}>R_{1}\left(m_{1}\right)$ and $C_{1} R^{p-q_{1}}<R_{2}\left(m_{2}\right)$. Then, (8) has a positive solution $w$ satisfying $m_{1}<\|w\|_{\infty}<m_{2}$ (resp., $m_{2}<\|w\|_{\infty}<m_{1}$ ).

Theorem 4. Assume that $(F 0)^{\prime}$ and (F5) and that there exist $R \in\left(0, R_{0}\right), m_{1}, m_{2}$ and $M_{1}$ (resp., $M_{2}$ ) such that $0<m_{1}<m_{2}<M_{1}$ (resp., $\left.0<m_{2}<m_{1}<M_{2}\right), C_{2} R^{p-q_{2}}>R_{1}\left(m_{1}\right), C_{1} R^{p-q_{1}}<R_{2}\left(m_{2}\right)$, and 
$C_{2} R^{p-q_{2}}>R_{1}\left(M_{1}\right)$ (resp., $C_{1} R^{p-q_{1}}<R_{2}\left(M_{2}\right)$ ). Then, (8) has two positive solutions $w_{1}$, $w_{2}$ satisfying $m_{1}<\left\|w_{1}\right\|_{\infty}<m_{2}<\left\|w_{2}\right\|_{\infty}<M_{1}$ (resp., $\left.m_{2}<\left\|w_{1}\right\|_{\infty}<m_{1}<\left\|w_{2}\right\|_{\infty}<M_{2}\right)$.

Theorem 5. Assume that (F0)' and (F5) hold and that there exist $R \in\left(0, R_{0}\right), m_{1}, m_{2}, M_{1}$ and $M_{2}$ such that $0<m_{2}<m_{1}<M_{2}<M_{1}$ (resp., $\left.0<m_{1}<m_{2}<M_{1}<M_{2}\right), C_{2} R^{p-q_{2}}>R_{1}\left(m_{1}\right), C_{2} R^{p-q_{2}}>R_{1}\left(M_{1}\right)$, $C_{1} R^{p-q_{1}}<R_{2}\left(M_{2}\right)$, and $C_{1} R^{p-q_{1}}<R_{2}\left(m_{2}\right)$. Then, (8) has three positive solutions $w_{1}, w_{2}, w_{3}$ satisfying $m_{2}<\left\|w_{1}\right\|_{\infty}<m_{1}<\left\|w_{2}\right\|_{\infty}<M_{2}<\left\|w_{3}\right\|_{\infty}<M_{1}$ (resp., $m_{1}<\left\|w_{1}\right\|_{\infty}<m_{2}<\left\|w_{2}\right\|_{\infty}<M_{1}<$ $\left.\left\|w_{3}\right\|_{\infty}<M_{2}\right)$.

Theorem 6. Assume that $(F 0)^{\prime}$ and (F5) hold and that $f_{0}=f_{\infty}=0$. Then, there exists $R^{*}>0$ such that for any $R \in\left(0, R^{*}\right)$, (8) has two positive solutions.

Proof. From $f_{0}=f_{\infty}=0$, it follows that $\lim _{m \rightarrow 0^{+}} R_{1}(m)=\lim _{m \rightarrow \infty} R_{1}(m)=\infty$. Then, there exists $m_{1}^{*} \in \mathbb{R}_{+}$ satisfying $R_{1}\left(m_{1}^{*}\right)=\min \left\{R_{1}(m): m \in \mathbb{R}_{+}\right\} \in \mathbb{R}_{+}$. Set $R^{*}=\min \left\{R_{0},\left(\frac{R_{1}\left(m_{1}^{*}\right)}{C_{2}}\right)^{\frac{1}{p-q_{2}}}\right\}$. For any $R \in$ $\left(0, R^{*}\right)$, there exist $m_{1}=m_{1}(R)$ and $M_{1}=M_{1}(R)$ such that $0<m_{1}<m_{1}^{*}<M_{1}$ and $C_{2} R^{p-q_{2}}>$ $R_{1}\left(m_{1}\right)=R_{1}\left(M_{1}\right)$. By Lemma 3,

$$
\text { for any } R \in\left(0, R^{*}\right), i\left(T_{R}, \mathcal{K}_{m}, \mathcal{K}\right)=0 \text { for } m \in\left\{m_{1}, M_{1}\right\} \text {. }
$$

On the other hand, since $f_{0}=f_{\infty}=0, \lim _{m \rightarrow 0^{+}} R_{2}(m)=\lim _{m \rightarrow \infty} R_{2}(m)=\infty$. For any $R \in\left(0, R^{*}\right)$, there exist $m_{1}^{\prime} \in\left(0, m_{1}\right)$ and $M_{1}^{\prime} \in\left(M_{1}, \infty\right)$ such that $C_{1} R^{p-q_{1}}<R_{2}\left(m_{1}^{\prime}\right)=R_{2}\left(M_{1}^{\prime}\right)$. By Lemma 4,

$$
\text { for any } R \in\left(0, R^{*}\right), i\left(T_{R}, \mathcal{K}_{m}, \mathcal{K}\right)=1 \text { for } m \in\left\{m_{1}^{\prime}, M_{1}^{\prime}\right\}
$$

Then, by (14) and (15) and the additivity property of the fixed point index, for any $R \in\left(0, R^{*}\right)$,

$$
i\left(T_{R}, \mathcal{K}_{m_{1}} \backslash \overline{\mathcal{K}}_{m_{1}^{\prime}}, \mathcal{K}\right)=-1 \text { and } i\left(T_{R}, \mathcal{K}_{M_{1}^{\prime}} \backslash \overline{\mathcal{K}}_{M_{1}}, \mathcal{K}\right)=1
$$

In view of the solution property of the fixed point index, for any $R \in\left(0, R^{*}\right)$, there exist $u_{1} \in$ $\mathcal{K}_{m_{1}} \backslash \overline{\mathcal{K}}_{m_{1}^{\prime}}$ and $u_{2} \in \mathcal{K}_{M_{1}^{\prime}} \backslash \overline{\mathcal{K}}_{M_{1}}$ such that $T_{R}\left(u_{i}\right)=u_{i}$ for $i=1$, 2. Thus, (8) has two positive solutions for any $R \in\left(0, R^{*}\right)$.

Note that if either (i) $f_{0}=0$ and $f_{\infty} \in \mathbb{R}_{+}$or $(i i) f_{0} \in \mathbb{R}_{+}$and $f_{\infty}=0$, then there exists $\epsilon>0$ satisfying $R_{1}(m)>\epsilon$ for all $m \in \mathbb{R}_{+}$. By an argument similar to those in the proof of Theorem 6 , we have the following theorem:

Theorem 7. Assume that (F0)' and (F5) hold and that either (i) $f_{0}=0$ and $f_{\infty} \in \mathbb{R}_{+}$or (ii) $f_{0} \in \mathbb{R}_{+}$and $f_{\infty}=0$. Then, there exists $R^{*}>0$ such that (8) has a positive solution for any $R \in\left(0, R^{*}\right)$.

If $f_{0}=0$ and $f_{\infty}=\infty$ (resp., $f_{0}=\infty$ and $f_{\infty}=0$ ), by Remark $4, \lim _{m \rightarrow+0} R_{2}(m)=\infty$ and $\lim _{m \rightarrow+\infty} R_{1}(m)=0$ (resp., $\lim _{m \rightarrow+0} R_{1}(m)=0$ and $\lim _{m \rightarrow+\infty} R_{2}(m)=\infty$ ). Then, for any $R \in \mathbb{R}_{+}$, there exist $m_{1}, m_{2}$ satisfying $0<m_{2}<m_{1}$ (resp., $\left.0<m_{1}<m_{2}\right), R_{2}\left(m_{2}\right)>C_{1} R^{p-q_{1}}$, and $R_{1}\left(m_{1}\right)<C_{2} R^{p-q_{2}}$. In view of Theorem 3 , we have the following theorem:

Theorem 8. Assume that (F0)' and (F5) hold and that either (i) $f_{0}=0$ and $f_{\infty}=\infty$ or (ii) $f_{0}=\infty$ and $f_{\infty}=0$. Then, (8) has a positive solution for all $R \in \mathbb{R}_{+}$.

In the results so far, we assumed that $f$ is positive for all $u>0$, since it always satisfies $(F 0)^{\prime}$. If we assume that $f$ has a positive falling zero instead of $(F 0)^{\prime}$, i.e., $f$ satisfies the following:

$(F 0)^{\prime \prime}$ there exists $\beta \in \mathbb{R}_{+}$such that $f(u)>0$ for $u \in(0, \beta)$ and $f(u)<0$ for $u \in(\beta, \infty)$, 
then $\lim _{m \rightarrow \beta^{-}} R_{2}(m)=\infty$, so that we can obtain results similar to Theorems 3-8 above as follows:

Theorem 9. Assume that $(F 0)^{\prime \prime}$ and (F5) and that there exist $R \in\left(0, R_{0}\right)$ and $m_{1}$ such that $0<m_{1}<\beta$ and $C_{2} R^{p-q_{2}}>R_{1}\left(m_{1}\right)$. Then, (8) has a positive solution w satisfying $m_{1}<\|w\|_{\infty}<\beta$.

Theorem 10. Assume that (F0)" and (F5) and that there exist $R \in\left(0, R_{0}\right), m_{1}$ and $m_{2}$ such that $0<m_{2}<$ $m_{1}<\beta, C_{2} R^{p-q_{2}}>R_{1}\left(m_{1}\right)$ and $C_{1} R^{p-q_{1}}<R_{2}\left(m_{2}\right)$. Then, (8) has two positive solutions $w_{1}$, $w_{2}$ satisfying $m_{2}<\left\|w_{1}\right\|_{\infty}<m_{1}<\left\|w_{2}\right\|_{\infty}<\beta$.

Theorem 11. Assume that (F0)" and (F5) hold and that there exist $R \in\left(0, R_{0}\right), m_{1}, m_{2}$ and $M_{1}$ such that $0<m_{1}<m_{2}<M_{1}<\beta, C_{2} R^{p-q_{2}}>R_{1}\left(m_{1}\right), C_{2} R^{p-q_{2}}>R_{1}\left(M_{1}\right)$, and $C_{1} R^{p-q_{1}}<R_{2}\left(m_{2}\right)$. Then, (8) has three positive solutions $w_{1}, w_{2}$, $w_{3}$ satisfying $m_{1}<\left\|w_{1}\right\|_{\infty}<m_{2}<\left\|w_{2}\right\|_{\infty}<M_{1}<\left\|w_{3}\right\|_{\infty}<\beta$.

Theorem 12. Assume that $(F 0)^{\prime \prime}$ and (F5) hold and that $f_{0}=0$. Then, there exists $R^{*}>0$ such that for any $R \in\left(0, R^{*}\right)$, (8) has two positive solutions.

Theorem 13. Assume that $(F 0)^{\prime \prime}$ and (F5) hold and that $f_{0} \in \mathbb{R}_{+}$. Then, there exists $R^{*}>0$ such that (8) has a positive solution for any $R \in\left(0, R^{*}\right)$.

Theorem 14. Assume that (F0)" and (F5) hold and that $f_{0}=\infty$. Then, (8) has a positive solution for all $R \in \mathbb{R}_{+}$.

Finally, the examples to illustrate the results obtained in this paper are given.

\section{Example 1.}

(1) Let $f(s)=\left\{\begin{array}{l}s^{\alpha_{1}}, \text { for } s \in[0,1), \\ s^{\alpha_{2}}, \text { for } s \in[1, \infty),\end{array}\right.$ and let $K(r)=r^{-q}$ for $r \in \mathbb{R}_{+}$, where $p \in(1, N), q>p$, and $0<\alpha_{2}<p-1<\alpha_{1}$. Then, $(F 0)^{\prime},(F 2)$ (or $\left.(F 2)^{\prime}\right),(F 5)$, and $f_{0}=f_{\infty}=0$ are satisfied. By Theorem 1 (or Theorem 2) and Theorem 6, there exist positive constants $R_{*}$ and $R^{*}$ such that (1) has two positive solutions for $R \in\left(0, R^{*}\right)$, and it has no nontrivial solutions for $R>R_{*}$.

(2) Let $K(r)=r^{-3}$ for $r \in \mathbb{R}_{+}$, and let $p=N-1=2$. Then, in the assumption (F5) and in (9), $q_{1}=q_{2}=3, C_{1}=C_{2}=1, R_{0}=\infty$, and $k_{1}(t)=k_{2}(t)=t^{-1} \in \mathcal{C} \backslash L^{1}(0,1)$. By direct calculation, $A_{1}=\frac{1}{4}(1-\ln 2)$ and $A_{2}=\frac{1}{2}$. Let:

$$
f(s)= \begin{cases}s^{2}, & \text { for } s \in\left[0,2^{4} A_{1}^{2}\right) \\ 2^{6} A_{1}^{3} s^{\frac{1}{2}}, & \text { for } s \in\left[2^{4} A_{1}^{2}, 2^{8}\right) \\ 2^{-6} A_{1}^{3} s^{2}, & \text { for } s \in\left[2^{8}, \infty\right)\end{cases}
$$

Then, $f_{0}=0$ and $f_{\infty}=\infty$. Thus, by Remark 4, $\lim _{m \rightarrow+0} R_{i}(m)=\infty$ and $\lim _{m \rightarrow+\infty} R_{i}(m)=0$ for $i=1,2$. Moreover, since $\bar{f}(m)=f(m)$ and $f(m)=f(m / 4), R_{2}(m)$ (resp., $R_{1}(m)$ ) is decreasing in $\left(0,2^{4} A_{1}^{2}\right)$ (resp., $\left.\left(0,2^{6} A_{1}^{2}\right)\right)$, increasing in $\left(2^{4} A_{1}^{2}, 2^{8}\right)$ (resp., $\left.\left(2^{6} A_{1}^{2}, 2^{10}\right)\right)$, and decreasing in $\left(2^{8}, \infty\right)$ (resp., $\left.\left(2^{10}, \infty\right)\right)$. Since $R_{1}\left(2^{6} A_{1}^{2}\right)=\left(4 A_{1}^{3}\right)^{-1}<\left(2 A_{1}^{3}\right)^{-1}=R_{2}\left(2^{8}\right)$, for each $R \in\left(2 A_{1}^{3}, 4 A_{1}^{3}\right)$, there exist $m_{2}<2^{4} A_{1}^{2}<m_{1}<$ $2^{6} A_{1}^{2}<M_{2}<2^{8}<M_{1}$ satisfying $R^{-1}<R_{2}\left(m_{2}\right), R^{-1}>R_{1}\left(m_{1}\right), R^{-1}<R_{2}\left(M_{2}\right)$ and $R^{-1}>R_{1}\left(M_{1}\right)$. Consequently, by Theorem 5 and Theorem 8, Problem (1) has three positive solutions for $R \in\left(2 A_{1}^{3}, 4 A_{1}^{3}\right)$, and it has a positive solution for all $R \in \mathbb{R}_{+}$.

Author Contributions: All authors contributed equally to the manuscript and read and approved the final draft.

Funding: This research was supported by the Basic Science Research Program through the National Research Foundation of Korea (NRF) funded by the Ministry of Education (2017R1D1A1B03035623). 
Acknowledgments: The authors would like to thank the anonymous reviewers for their useful suggestions.

Conflicts of Interest: The authors declare no conflict of interest.

\section{References}

1. Tanaka, S. Morse index and symmetry-breaking for positive solutions of one-dimensional Hénon type equations. J. Differ. Equ. 2013, 255, 1709-1733. [CrossRef]

2. Shivaji, R.; Sim, I.; Son, B. A uniqueness result for a semipositone $p$-Laplacian problem on the exterior of a ball. J. Math. Anal. Appl. 2017, 445, 459-475. [CrossRef]

3. Drábek, P.; Ho, K.; Sarkar, A. The Fredholm alternative for the $p$-Laplacian in exterior domains. Nonlinear Anal. 2018, 174, 17-53. [CrossRef]

4. Agarwal, R.P.; Lü, H.; O’Regan, D. Eigenvalues and the one-dimensional p-Laplacian. J. Math. Anal. Appl. 2002, 266, 383-400. [CrossRef]

5. An, Y.; Kim, C.-G.; Shi, J. Exact multiplicity of positive solutions for a $p$-Laplacian equation with positive convex nonlinearity. J. Differ. Equ. 2016, 260, 2091-2118. [CrossRef]

6. Kajikiya, R.; Lee, Y.-H.; Sim, I. One-dimensional $p$-Laplacian with a strong singular indefinite weight. I. Eigenvalue. J. Differ. Equ. 2008, 244, 1985-2019. [CrossRef]

7. Korman, P. Existence and uniqueness of solutions for a class of $p$-Laplace equations on a ball. Adv. Nonlinear Stud. 2011, 11, 875-888. [CrossRef]

8. Mohammed, A. Positive solutions of the $p$-Laplace equation with singular nonlinearity. J. Math. Anal. Appl. 2009, 352, 234-245. [CrossRef]

9. Ouyang, T.; Shi, J. Exact multiplicity of positive solutions for a class of semilinear problems. J. Differ. Equ. 1998, 146, 121-156. [CrossRef]

10. Ouyang, T.; Shi, J. Exact multiplicity of positive solutions for a class of semilinear problem. II. J. Differ. Equ. 1999, 158, 94-151. [CrossRef]

11. Papageorgiou, N.S.; Vetro, C.; Vetro, F. Existence of positive solutions for nonlinear Dirichlet problems with gradient dependence and arbitrary growth. Electron. J. Qual. Theory Differ. Equ. 2018, 18, 1-9. [CrossRef]

12. Reichel, W.; Walter, W. Radial solutions of equations and inequalities involving the $p$-Laplacian. J. Inequal. Appl. 1997, 1, 47-71. [CrossRef]

13. Chhetri, M.; Girg, P. Nonexistence of nonnegative solutions for a class of $(p-1)$-superhomogeneous semipositone problems. J. Math. Anal. Appl. 2006, 322, 957-963. [CrossRef]

14. Allegretto, W.; Huang, Y.X. Principal eigenvalues and Sturm comparison via Picone's identity. J. Differ. Equ. 1999, 156, 427-438. [CrossRef]

15. Chhetri, M.; Shivaji, R.; Son, B.; Sankar, L. An existence result for superlinear semipositone $p$-Laplacian systems on the exterior of a ball. Differ. Integr. Equ. 2018, 31, 643-656.

16. Iaia, J.A. Existence and nonexistence of solutions for semilinear equations on exterior domains. Electron. J. Differ. Equ. 2016, 227, 1-9.

17. Iaia, J.A. Existence for semilinear equations on exterior domains. Electron. J. Qual. Theory Differ. Equ. 2016, 108, 1-12. [CrossRef]

18. Iaia, J.A. Existence and nonexistence of solutions for sublinear equations on exterior domains. Electron. J. Differ. Equ. 2017, 214, 1-12.

19. Iaia, J.A. Existence of solutions for semilinear problems with prescribed number of zeros on exterior domains. J. Math. Anal. Appl. 2017, 446, 591-604. [CrossRef]

20. Joshi, J.; Iaia, J. Existence of solutions for similinear problems with prescribed number of zeros on exterior domains. Electron. J. Differ. Equ. 2016, 112, 1-11.

21. Ko, E.; Lee, E.K.; Shivaji, R. Multiplicity results for classes of singular problems on an exterior domain. Discret. Contin. Dyn. Syst. 2013, 33, 5153-5166. [CrossRef]

22. Morris, Q.; Shivaji, R.; Sim, I. Existence of positive radial solutions for a superlinear semipositone $p$-Laplacian problem on the exterior of a ball. Proc. R. Soc. Edinb. Sect. A 2018, 148, 409-428. [CrossRef]

23. Stegliński, R. Remarks on the existence of infinitely many solutions for a $p$-Laplacian equation involving oscillatory nonlinearities. Electron. J. Qual. Theory Differ. Equ. 2017, 56, 1-12. [CrossRef]

24. Joshi, J. Existence and nonexistence of solutions for sublinear problems with prescribed number of zeros on exterior domains. Electron. J. Differ. Equ. 2017, 133, 1-10. 
25. Byun, J.; Sim, I. A relation between two classes of indefinite weights in singular one-dimensional $p$-Laplacian problems. Math. Inequal. Appl. 2007, 10, 889-894. [CrossRef]

26. Wang, J. The existence of positive solutions for the one-dimensional p-Laplacian. Proc. Am. Math. Soc. 1997, 125, 2275-2283. [CrossRef]

27. Deimling, K. Nonlinear Functional Analysis; Springer: Berlin, Germany, 1985.

28. Guo, D.J.; Lakshmikantham, V. Nonlinear Problems in Abstract Cones; Notes and Reports in Mathematics in Science and Engineering; Academic Press, Inc.: Boston, MA, USA, 1988.

(C) 2019 by the authors. Licensee MDPI, Basel, Switzerland. This article is an open access article distributed under the terms and conditions of the Creative Commons Attribution (CC BY) license (http:/ / creativecommons.org/licenses/by/4.0/). 